\title{
Original
}

\section{Anemia materna en el tercer trimestre de embarazo como factor de riesgo para parto pretérmino}

\author{
(Maternal Anemia During the Third Trimester of \\ Pregnancy as a Risk Factor for Preterm Labor)
}

\author{
Loretta Giacomin-Carmiol, Manrique Leal-Mateos, Rafael Ángel Moya-Sibaja
}

\section{Resumen}

Objetivos: Analizar la relación entre la anemia y el parto pretérmino en el Servicio de Obstetricia del Hospital "Dr. Rafael Ángel Calderón Guardia”, durante 2006.

Materiales y métodos: Estudio de 110 pacientes y 220 controles. Se utilizó el registro estadístico de la sala de partos del Servicio de Obstetricia para la identificación de la muestra. Los criterios de inclusión para el grupo de casos fueron: edad materna mayor o igual a 18 años, edad materna menor de 35 años, primigesta, embarazo mayor de 32 semanas, embarazo único, y recién nacido vivo al momento del parto. Los criterios de exclusión fueron: embarazo complicado por enfermedades médicas crónicas o inducidas por este, antecedente en la historia clínica de sangrado transvaginal durante el primer o segundo trimestre, antecedente en la historia clínica de síntomas que sugirieran infección del tracto urinario o de otro sistema, antecedente en la historia clínica del uso de tabaco, alcohol u otra sustancia ilícita, productos con algún tipo de malformación diagnosticada previo a la labor del parto o durante el nacimiento, parto inducido o cesárea electiva, parto atendido de forma extrahospitalaria, ausencia o registro incompleto de alguna variable de interés. Las pacientes del grupo de control fueron aquellas internadas durante el mismo periodo. Los dos controles para cada caso fueron seleccionados de forma aleatoria, pareados por grupo quinquenal de edad y fecha de la atención del parto. Los criterios de inclusión y exclusión fueron los mismos aplicados al grupo de casos. Se definió como anemia una hemoglobina menor a $11 \mathrm{~g} / \mathrm{dl}$. La magnitud de la asociación entre la anemia y el parto pretérmino se evaluó mediante la razón de probabilidades (Odds Ratio)

Resultados: La hemoglobina promedio de los casos fue de $12,3 \mathrm{~g} / \mathrm{dl}(\mathrm{DS} \pm 1,2)$ y la de los controles de 12,9 g/dl (DS $\pm 1,1 ; \mathrm{p}<0,001)$. El hematocrito promedio de los casos fue de 36,2 $\%(\mathrm{DS} \pm 3,6)$ y la de los controles de 38,2 (DS $\pm 3,1 ; \mathrm{p}<0,001)$. El porcentaje de pacientes con anemia en el grupo de casos fue del 10,9\% $(n=12)$ y en el grupo control del $4,1 \%(n=9) ;(p=0,016)$. La razón de probabilidades mostró una asociación positiva entre la presencia de anemia y un

Servicio de Obstetricia, Hospital Dr. Rafael Ángel Calderón Guardia

Abreviaturas: C.C.S.S., Caja Costarricense de Seguro Social; Hospital Dr. Rafael Ángel Calderón Guardia, HCG.

Correspondencia:

Manrique Leal Mateos; manrique.leal@gmail.com parto pretérmino de 2,87 (IC 95\% 1.08-7,69).

Conclusiones: Se demostró una asociación entre la anemia en el tercer trimestre y parto de pretérmino.

Descriptores: Anemia, parto pretérmino, Hospital Calderón Guardia 


\section{Abstract}

Objective: To explore the relationship between anemia and preterm birth at the Obstetrics Service of the Rafael Angel Calderón Guardia Hospital during the year 2006.

Methods: A Case and Control study was made obtaining the sample from the statistical base of the Labor and Delivery Ward. There were 110 cases and 220 controls.Inclusion criteria were: age between 18 and 35 years, first pregnancy, pregnancy of more than 32 weeks, single pregnancy and live born. Exclusion criteria were: pregnancy complicated by a chronic medical illness or acquired during pregnancy, history of vaginal bleeding during pregnancy, history of urinary tract infection or of other system, alcohol or drug abuse, presence of fetal malformation, induced delivery or elective cesarean section, extrahospitalary delivery and absence or incomplete information. The patients from the control group were admitted to the hospital in the same period of time. Two controls were selected randomly, pared by quinquenal group of age and delivery date. The inclusion and exclusion criteria were the same as in the case group. Anemia was defined as hemoglobin less than $11 \mathrm{~g} / \mathrm{dl}$. The magnitude of the association between anemia and preterm birth was evaluated by the odds ratio.

Results: The mean hemoglobin value of the cases was $12.3 \mathrm{~g} / \mathrm{dl}$ (DS + 1.2) versus $12.9 \mathrm{~g} / \mathrm{dl}$ (DS +1.1 ) in the control group. The mean hematocrit of the cases was $36.2 \%$ (DS +3.6 ) and $38.2 \%$ $(\mathrm{DS}+3.1)(\mathrm{p}<0.001)$ in the control group. The percentage of patients with anemia in the control group was $10.9 \%(n=12)$ and $4.1 \%(n=9)$ in the control group $(\mathrm{p}<0.016)$. The odds ratio showed a positive association between anemia and preterm birth (2.87; CI 95\% 1.08-7.69).

Conclusion: The present study demonstrated an association between anemia and preterm birth during the third trimester.

Key words: Anemia, preterm birth and Rafael Angel Calderón Guardia Hospital

Recibido: 25 de agosto de 2008 Aceptado: 21 de octubre de 2008

El parto pretérmino es una de las principales causas de morbilidad y mortalidad perinatal en nuestro país y en el nivel mundial. ${ }^{1}$

Muchos son los factores de riesgo propios del embarazo que han sido asociados al nacimiento de productos de forma prematura. Igualmente, son numerosas las características maternas que se han relacionado con la prematuridad. Sin embargo, dado que la etiología del parto prematuro es en ocasiones multifactorial, establecer una relación causal única estricta resulta a veces difícil. ${ }^{2-5}$

Dentro de los factores de riesgo maternos, la anemia ha sido uno de los que más controversia ha causado. A pesar de la evidencia mostrada desde hace varios años, algunos autores dudan de una relación causal directa entre esta y el parto prematuro. ${ }^{6-10}$

Dada la controversia mostrada en relación con el tema y la falta de evidencia nacional sobre esta asociación, el presente estudio tiene como propósito explorar con detalle el vínculo entre la anemia durante el tercer trimestre y el parto pretérmino.

\section{Materiales y métodos}

Se realizó un estudio de casos y controles en el Servicio de Obstetricia del Hospital "Dr. Rafael Ángel Calderón Guardia" (HCG), que comprendió el análisis retrospectivo de la información contenida en el expediente clínico de las pacientes que tuvieron su parto durante 2006. El estudio fue aprobado por el Comité de Ética del Hospital.

La muestra fue calculada con el programa Statcalc de EpiInfo 2002, con un $\alpha=0,05$ y un $\beta=0,20$, una relación caso control 1:2 y una prevalencia esperada máxima de anemia en el grupo de parto pretérmino del $50 \%$. Con base en esto se estableció un número de casos de 110 y de controles de 220 para obtener, de ser así, una diferencia estadísticamente significativa entre los grupos.

Se utilizó el registro estadístico de la sala de partos del Servicio de Obstetricia para la identificación de la muestra. De él se tomo el número de expediente de las pacientes seleccionadas para después revisar cada una de las historias clínicas contenidas en él al momento del ingreso al Hospital. 
Mediante un muestreo consecutivo se fueron identificando cada uno de los casos. Se revisaron los datos de las pacientes a partir del 1 de enero de 2006 y hasta el 29 de noviembre de 2006 (momento cuando se completo el tamaño de la muestra requerida).

Con el objetivo de obtener la mejor estimación posible de la asociación de interés, se empleó el método de restricción para el control de variables que pudieran influir y confundir los resultados obtenidos. Para esto se utilizaron criterios de inclusión y exclusión.

Los criterios de inclusión para identificar las pacientes que conformaron el grupo de casos fueron los siguientes: edad materna mayor o igual a 18 años y menor de 35 años, primigesta, embarazo mayor de 32 semanas de gestación, embarazo único y recién nacido vivo al momento del parto.

Los criterios de exclusión aplicados a las pacientes seleccionadas fueron: embarazo complicado por enfermedades médicas crónicas o inducidas por este, antecedente en la historia clínica de sangrado transvaginal durante el primer o segundo trimestre, antecedente en la historia clínica de síntomas que sugirieran infección del tracto urinario o de otro sistema, antecedente en la historia clínica del uso de tabaco, alcohol u otra sustancia ilícita, productos con algún tipo de malformación diagnosticada previo a la labor del parto o durante el nacimiento, parto inducido o cesárea electiva, parto atendido de forma extrahospitalaria, ausencia o registro incompleto de alguna variable de interés.

Las pacientes que conformaron el grupo de control fueron aquellas con un embarazo mayor o igual a 37 semanas, internadas en el Servicio de Obstetricia para la atención de su labor de parto durante el mismo periodo. Los dos controles para cada caso fueron seleccionados de forma aleatoria, pareados por grupo quinquenal de edad y fecha de la atención. Los criterios de inclusión y exclusión para este grupo fueron los mismos aplicados al grupo de casos.

Para el análisis se utilizaron los resultados del hemograma obtenido al momento del ingreso de la paciente al Hospital para la atención de su labor de parto. Se definió como anemia una hemoglobina menor a $11 \mathrm{~g} / \mathrm{dl} .{ }^{11}$

Se definió como parto pretérmino aquel que ocurrió antes de las 37 semanas de gestación cumplidas con base en la fecha de última regla reportada por la paciente o según ultrasonido realizado durante el primer trimestre del embarazo.

Para la recolección de los datos se utilizó una hoja electrónica elaborada para el estudio.

Se utilizó el programa EpiInfo 2002 para el procesamiento de la información. Las variables cualitativas se analizaron mediante frecuencias $\mathrm{y}$ proporciones. Los resultados derivados de las variables cuantitativas se expresaron con medidas de tendencia central y de dispersión. La relación entre variables cualitativas se realizó con la prueba estadística de Chi cuadrada $\left(\mathrm{x}^{2}\right)$. La diferencia de promedios entre dos grupos se comparó por medio de la prueba de t Student.

La magnitud de la asociación entre la anemia y el parto pretérmino se evaluó mediante la razón de probabilidades (Odds Ratio en inglés) que indica la cantidad de veces que es más probable encontrar la enfermedad (parto pretérmino) en el grupo de pacientes expuestas al factor de riesgo (anemia). La razón de probabilidades (OR) de 1,0 indica que no existe relación entre las variables y si es mayor a este valor, que sí la hay.

\section{Resultados}

Se excluyeron del análisis un total de 112 casos: 48 por presentar enfermedades crónicas o inducidas por el embarazo, 40 por presentar alguna indicación para la inducción de su labor de parto o por ser cesárea electiva, 11 por presentar algún tipo de infección al ingreso hospitalario, 11 por presentar productos con algún tipo de malformación, 1 por toxicomanía y 1 por ser un parto extrahospitalario.

La edad promedio de los casos fue de 22,7 años (DS+ $4,7)$ y la de los controles de 22,1 años $\left(\mathrm{DS}_{ \pm} \pm 4,1\right)$. No hubo una diferencia estadísticamente significativa entre los grupos, dado el apareamiento de la muestra $(p=0,24)$. La edad gestacional promedio de los casos fue de 34,6 años $(\mathrm{DS} \pm 2,3)$ y la de los controles, de 39,2 años $(\mathrm{DS} \pm 1,2)$ (Cuadro 1).

La hemoglobina promedio de los casos fue de $12,3 \mathrm{~g} / \mathrm{dl}$ $(\mathrm{DS}+1,2)$ y la de los controles, de $12,9 \mathrm{~g} / \mathrm{dl}(\mathrm{DS}+1,1)$. Esta diferencia fue estadísticamente significativa $(\mathrm{p}<0,001)$. El hematocrito promedio de los casos fue de 36,2 \%(DS $\pm 3,6$ ) y la de los controles, de 38,2 (DS+3,1). Esta diferencia también fue estadísticamente significativa $(\mathrm{p}<0,001)$ (Figuras 1 y 2).

Cuadro 1. Distribución de principales variables
analizadas. Estudio sobre anemia como factor
de riesgo para parto pretérmino, HCG, 2006.

\begin{tabular}{|llll|}
\hline & Casos & Controles & Valor de $\mathrm{p}$ \\
\hline $\begin{array}{l}\text { Edad } \\
\text { (promedio) }\end{array}$ & 22,7 años & 22,1 años & $\mathrm{p}=0,24$ \\
EG & $34,6 \mathrm{sem}$ & $39,2 \mathrm{sem}$ & - \\
Hemoglobina & $12,3 \mathrm{~g} / \mathrm{dl}$ & $12,9 \mathrm{~g} / \mathrm{dl}$ & $\mathrm{p}<0.001$ \\
Hematocrito & $36,2 \%$ & $38,2 \%$ & $\mathrm{p}<0.001$ \\
Anemia (\%) & $10.9 \%$ & $4.1 \%$ & $\mathrm{p}=0.016$ \\
\hline
\end{tabular}




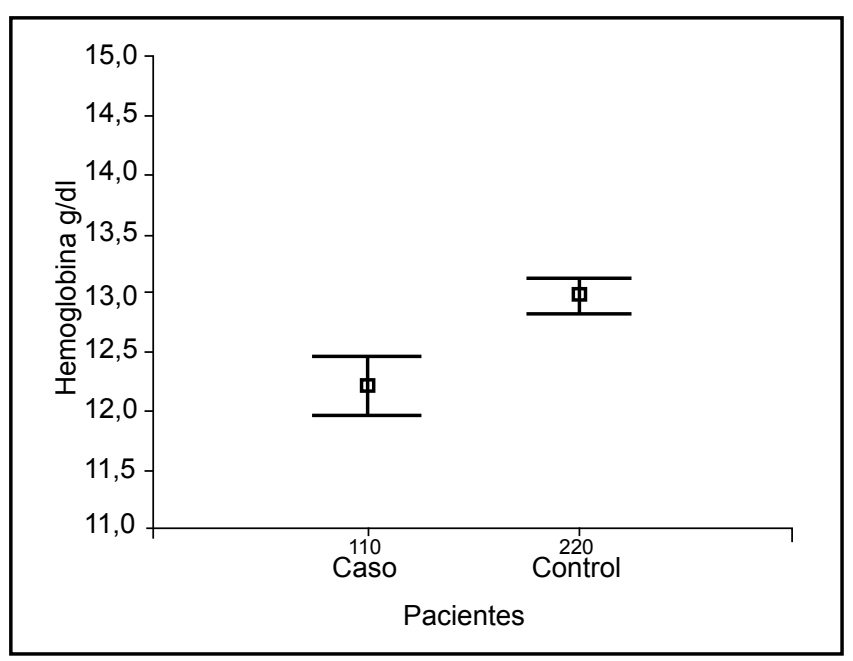

Figura 1. Hemogloina promedio. Estudio sobre anemia como factor de riesgo para el parto pretérmino, HCG, 2006.

El porcentaje de pacientes con anemia en el grupo de casos fue del $10,9 \%(n=12)$ y en el grupo control, del $4,1 \%$ $(n=9)$. Esta diferencia fue estadísticamente significativa $(\mathrm{p}=0,016)$. La razón de probabilidades $(\mathrm{OR})$ mostró una asociación positiva entre la presencia de anemia y la presentación de un parto pretérmino de 2,87 (IC 95\% 1.087,69) (Cuadro 2).

\section{Discusión}

La anemia es un problema de salud pública generalizado que tiene consecuencias de gran alcance para la salud humana y para el desarrollo social y económico. A pesar de que los cálculos de la prevalencia de la anemia varían mucho y a menudo no hay datos exactos, puede suponerse que en países en vías de desarrollo una proporción considerable de niños y de mujeres en edad fértil padecen anemia. ${ }^{12}$

En América Latina la tasa promedio de anemia en mujeres no embarazadas se estima en el $20 \%$, con un rango del $8 \%$ al $35 \%{ }^{13}$ La prevalencia de anemia de las mujeres en edad fértil de Costa Rica semeja el promedio estimado en América Latina, con una prevalencia mucho menor de anemia severa. ${ }^{14}$

La anemia de mujeres en edad reproductiva hace suponer una prevalencia mayor de esta en mujeres embarazadas. Lo anterior, por tanto, hace que durante el embarazo esta patología se asocie a malos resultados perinatales, dentro de los que resalta el parto prematuro y el bajo peso al nacer, entre otros. ${ }^{12,15}$

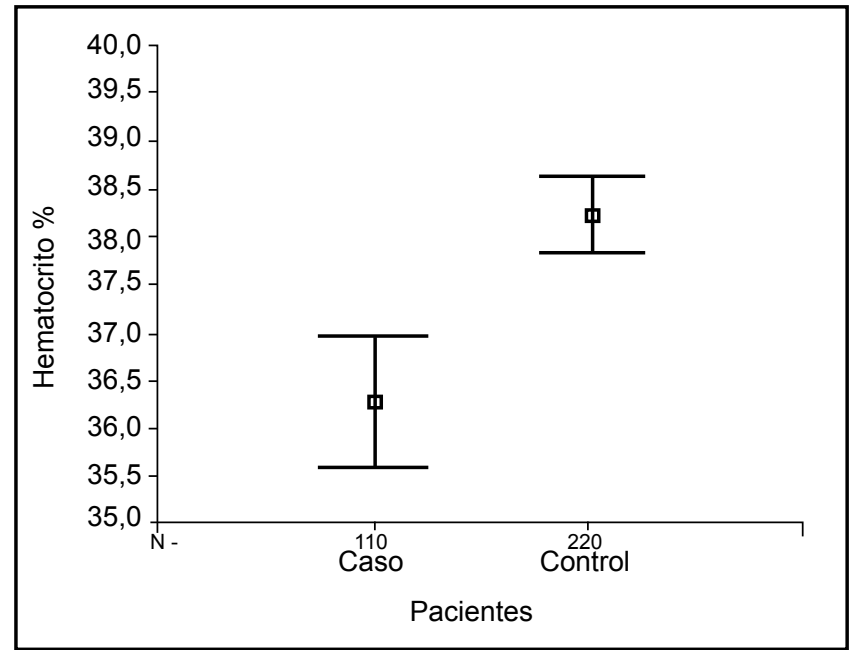

Figura 2. Hematocrito promedio. Estudio sobre la anemia como factor de riesgo para el parto pretermino, HCG, 2006

\begin{tabular}{|lccc|}
\multicolumn{3}{|c}{$\begin{array}{c}\text { Cuadro 2. Distribución de pacientes. Estudio } \\
\text { sobre anemia como factor de riesgo para parto } \\
\text { pretérmino, HCG, 2006. }\end{array}$} \\
\hline & Con anemia & Sin anemia & Total \\
\hline Casos & 12 & 96 & 110 \\
Controles & 9 & 210 & 220 \\
Total & 24 & 306 & 330
\end{tabular}

OR: 2.87 (IC 95\% 1.08 - 7.69)

Con base en tales antecedentes, el presente estudio confirma en este hospital la relación positiva entre la presencia de anemia y el nacimiento de un producto pretérmino. Si bien, mediante el diseño de esta investigación no se puede determinar una relación causal directa entre esta patología y la prematuridad, se coincide con lo observado en otras investigaciones similares.

Por otro lado, la anemia no tratada puede ser un indicador indirecto de pobre nutrición y mala salud. La desnutrición materna asociada a un bajo estado socioeconómico ha sido vinculada también a resultados perinatales adversos y en especial a la prematuridad. ${ }^{16}$

En virtud de los factores discutidos, se recomienda el tratamiento enérgico de la anemia en mujeres de edad fértil, para con ello disminuir la prevalencia de esta patología en pacientes embarazadas y los posibles eventos adversos asociados a ella. 


\section{Referencias}

1. Ministerio de Salud. Informe anual del Sistema Nacional de Análisis de la Mortalidad Infantil en Costa Rica. San José, CR.: Ministerio de Salud; 2003: 14-16.

2. Blanco J. Clinical problems of preterm labor. Clin Obstet Gynecol 2000; 43: 713-716.

3. Berkowitz GS. An epidemiology study of preterm deleviry. Am J Epidemiol 1981; 113: 81-92.

4. Goldenberg RL, Iams JD, Mercer BM, Meis PJ, Moawad AH, Cooper RL et al. The preterm prediction study: The value of new vs standard risk factors in predicting early and all spontaneous preterm birth. Am J Public Health 1998; 88: 233- 238.

5. Pschirrer ER, Munga M. Risk factors for preterm labor. Clin Obstet Gynecol 2000; 43: 727-734.

6. Lieberman E, Ryan K, Monson R, Shoenbaum S. Association of maternal hematocrit with premature labor. Am J Obstet Gynecol 1988; 159: 107-114.

7. Klebanoff MA, Shiono PH, Selby JV, Trachtenberg AI, Graubard MA. Anemia and spontaneous preterm birth. Am J Obstet Gynecol 1991; 164: 59-63.

8. Scholl TO, Hediger ML, Fisher RL, Shearer J. Anemia vs iron deficiency: Increased risk of preterm delivery in a prospective study. Am J Clin Nutr 1992; 55: 985-988.
9. Klebanoff MA, Shiono PH, Berendes HW, Rhoads G. Facts and artifacts about anemia and preterm delivery. JAMA 1989; 262: 511515.

10. Scholl T, Reilly T. Anemia, iron and pregnancy outcome. J Nutr 2000; 130: 443S-447S.

11. Centers for Disease Control. CDC Criteria for anemia in children and childbearing-aged women. MMWR 1989; 38: 400-404.

12. Organización Mundial de la Salud. La anemia como centro de atención: Hacia un enfoque integrado para el control eficaz de la anemia. Declaración conjunta de la Organización Mundial de la Salud y el Fondo de las Naciones Unidas para la Infancia. Ginebra: OMS; 2004.

13. Mora JO, Mora OL. Anemia ferropriva. En: Mora JO, Mora OL. Deficiencia de micronutrientes en América Latina y el Caribe. Wahington, D.C.: OPS, OMS, USAID, Roche, OMNI; 1999. p.1-25.

14. Ministerio de Salud. Encuesta Nacional de Nutrición: Micronutrientes. San José, CR: Ministerio de Salud; 1996.

15. Scholl TO, Hediger ML. Anemia and iron deficiency anemia: Compilation of data on pregnancy outcome. Am J Clin Nutr 1994; 59(suppl): 492S-500S.

16. Weck R, Paulose T, Flaws JA. Environmental factors and poverty on pregnancy outcome. Clin Obstet Gynecol 2008; 51: 349-359.

\section{Comité Editorial Acta Médica Costarricense}

\section{Lista de revisión para autores}

Por favor, complete esta lista antes de enviar su manuscrito.

$\square$ Carta de presentación

Tres copias completas del manuscrito con cuadros y figuras, y en formato digital

Página de título

口 Título

$\square$ Título corto, no mayor de 45 caracteres

$\square$ Autor(es) y afiliación(es)

$\square$ Lugar donde se realizó el trabajo

$\square$ Descriptores en español e inglés

$\square$ Abreviaturas

$\square$ Dirección, teléfono y número de fax del autor a quien se debe dirigir toda correspondencia

$\square$ Fuentes de apoyo y manifestación de conflictos de interés
Artículo (a doble espacio)

$\square$ Carta de aprobación del estudio por Comité Ético - científico

$\square$ Resumen estructurado en español

$\square$ Resumen estructurado en inglés

$\square$ Introducción

$\square$ Materiales y métodos

$\square$ Resultados

$\square$ Discusión

$\square$ Agradecimientos y colaboradores

$\square$ Referencias

$\square$ Cuadros, leyendas de las figuras, figuras

$\square$ Permiso para reproducir todo material previamente publicado y autorización de pacientes para publicar fotografías

$\square$ Carta de cada autor de asignación de derechos, responsabilidad de participación y declaración de financiamiento, si existiera (puede ser una, firmada por todos los autores). 